\title{
Uso y manejo de las TIC como competencia genérica en estudiantes preuniversitarios
}

\section{Use and management of ICT as a generic competence of pre-university students}

\author{
Enrique Arturo Vázquez Uscanga 1, Juan Carlos Bernal Pinacho 1, Claudia Catalina Mendizábal Benítez2 \\ enriquevaus@gmail.com,bernalln@hotmail.com,cc.mendizabal@outlook.com \\ Universidad Veracruzana \\ México
}

\begin{abstract}
Resumen- La presente ponencia muestra los resultados de investigación en la cual se describió de manera comparativa el uso y manejo de las TIC como competencia genérica de 555 estudiantes del último año de tres planteles educativos adscritos al Sistema Nacional de Bachillerato (SNB) de la ciudad de Xalapa en México; cada plantel perteneciente a un subsistema de bachillerato: general, tecnológico y profesional-técnico, tomando en cuenta el uso y manejo de las TIC a partir de la Reforma Integral de la Educación Media Superior (RIEMS) implementada a nivel nacional en 2008. Se desarrolló un estudio cuantitativo de tipo descriptivo y transversal a través de la aplicación del cuestionario para estudiantes diseñado en el marco del proyecto Brecha Digital del Centro de Innovación e Investigación en Educación Superior de la Universidad Veracruzana, midiendo el uso y manejo de TIC de 547 estudiantes a través de dos saberes digitales: comunicación en entornos digitales y; literacidad digital. Los resultados de la investigación arrojaron que los estudiantes de los tres subsistemas de bachillerato saben comunicarse en entornos digitales en un nivel BajoMedio mientras que en el saber literacidad digital se ubicaron en el nivel Alto de cuatro niveles posibles (Bajo, Bajo-Medio, Medio-Alto y Alto).
\end{abstract}

Palabras clave: TIC, competencias genéricas, saberes digitales, educación media superior

Abstract- This paper shows the results of research which described in a comparative way the use and management of ICT as a generic competency of 555 students of the last year of three educational establishments attached to the National Baccalaureate System (SNB) of the city of Xalapa in Mexico; each campus belonging to a subsystem of baccalaureate: general, technological and professional-technical, taking into account the use and management of ICT from the Integral Reform of High School Education (RIEMS) implemented nationwide in 2008. It was developed a descriptive and transversal quantitative study through the application of the questionnaire for students designed within the framework of the Digital Divide project of the Center for Innovation and Research in Higher Education of the Universidad Veracruzana, measuring the use and ICT management of 555 students at through two digital knowledge: communication in digital environments and; digital literacy. The results of the research showed that the students of the three high school subsystems know how to communicate in digital environments at a Low-Medium level, while digital literacy skills were located at the High level of four possible levels (Low, Low-Medium, Medium-High and High).

Keywords: ICT, generic competences, digital knowledge, high school

\section{INTRODUCCIÓN}

La Educación Media Superior en México (EMS) de acuerdo con la Secretaría de Educación Pública (SEP, 2008) representa un periodo preuniversitario para dar respuesta de manera oportuna a los retos de la sociedad del conocimiento, los cambios sociales y económicos del país; bajo este contexto se implementó en 2008 la Reforma Integral a la Educación Media Superior (RIEMS) cuyo propósito fue incorporar al currículo un enfoque educativo centrado en el aprendizaje del estudiante y el uso intensivo de las TIC por parte de los estudiantes estableciendo a través de dicha reforma dos atributos de "Maneja las TIC para obtener información y expresar ideas" y "Utiliza las TIC para procesar e interpretar información" que forman parte de las competencias genéricas: "Se expresa y se comunica", y "Piensa crítica y reflexivamente" respectivamente. (SEP, 2008b: 3).

Con ello, la RIEMS ha pretendido establecer las competencias en el rubro de TIC que los estudiantes deben desarrollar a su paso por la EMS, a través de un mismo perfil de egreso y buscando impulsar el uso y manejo de las TIC para lograr a corto plazo la plena integración de sus egresados ya sea a la educación superior, o bien, a la vida laboral.

En este sentido, las competencias genéricas de acuerdo con SEP (2008) son comunes para todos los egresados de la EMS buscando que los estudiantes comprendan el mundo e influyan en él, aprendan de manera autónoma a lo largo de la vida, desarrollen relaciones armónicas con quienes les rodean y participen de manera eficaz en su vida social, profesional y política. Por su importancia y aplicaciones a lo largo de la vida, las competencias genéricas se identifican también como competencias clave; transversales, por ser necesarias para todas las disciplinas y espacios curriculares de la EMS; y transferibles, ya que permiten a los estudiantes de adquirir otras competencias.

En concordancia con Cervantes (2015) dentro de las competencias "se expresa y se comunica" y "piensa crítica y reflexivamente" se manifiestan atributos relacionados con el manejo de las TIC en los estudiantes, cuyo objetivo no es sólo su uso y apropiación como herramienta, sino la habilidad digital potencial que la herramienta le permite desarrollar, como lo es 
el procesamiento de la información y la comunicación en entornos digitales que le permitan contribuir a su entorno.

\section{CONTEXTO}

\section{A. Justificación}

La Secretaría de Educación del estado de Veracruz en México a través del Sistema Educativo Nacional dedica recursos económicos y humanos a la implementación de las TIC en los planteles educativos de nivel medio superior por lo que resulta pertinente indagar si dichos recursos dotan a los estudiantes de las competencias genéricas relacionadas con las TIC que establece la reforma y si los estudiantes logran un uso y manejo de las TIC similar al momento de egresar del bachillerato.

En este sentido, los resultados de esta investigación pueden ser útiles para que los directivos y actores educativos del estado de Veracruz en México definan estrategias relacionadas con el uso e implementación de las TIC que permitan fundamentar y establecer mejores medidas de gestión en los planteles de este nivel educativo para que sus estudiantes puedan lograr una apropiación tecnológica equitativa al momento de egresar de las aulas independientemente del subsistema de bachillerato que hayan cursado.

\section{B. Objetivos}

Con base en lo anterior, el objetivo general de la investigación consistió en analizar de manera comparativa el uso y manejo de TIC como competencia genérica en los estudiantes de tres planteles públicos adscritos al SNB Región Xalapa, Veracruz en México, cada uno perteneciente a un subsistema de bachillerato: general, tecnológico y técnicoprofesional para indagar si poseen un mismo nivel en cada uno de los subsistemas de bachillerato y contar con un diagnóstico que muestre qué es lo que saben los estudiantes de TIC, con qué intención y con qué frecuencia las usan.

Así también, se plantearon dos objetivos específicos que se relacionan con los dos atributos de competencias genéricas de la RIEMS: a) describir el nivel de comunicación en entornos digitales que presentan los estudiantes del SNB Región Xalapa, Veracruz para distinguir semejanzas y/o diferencias por subsistema de bachillerato y b) describir el nivel de literacidad digital de los estudiantes del SNB Región Xalapa, Veracruz para distinguir semejanzas y/o diferencias por subsistema de bachillerato.

\section{Marco teórico}

El componente teórico de este trabajo está enmarcado en tres conceptos principales que se abordaron desde la perspectiva de varios autores y definiendo una postura para esta investigación: competencia al ser el eje principal de la Reforma y sobre el cual se construye el perfil del egresado del SNB, saberes digitales y por último brecha digital para enmarcar las posibles diferencias del nivel de uso y manejo de TIC en los estudiantes de los diferentes subsistemas de bachillerato.

El concepto de competencia, tal y como se entiende en la educación, resulta de las nuevas teorías de cognición y básicamente significa saberes de ejecución. Puesto que todo conocer se traduce en un saber, entonces, es posible decir que son recíprocos competencia y saber: saber pensar, saber desempeñar, saber interpretar, saber actuar en diferentes escenarios, desde sí y para la sociedad (dentro de un contexto determinado).

En este sentido, Tovar y Serna (2013) distinguen cuatro grados de alcance en las competencias durante la educación: 1) competencia genérica o básica definida como la aptitud útil para cualquier actividad, 2) competencia disciplinar como la capacidad que corresponde a una disciplina o área curricular, 3) competencia determinada o técnica entendida como la capacidad necesaria para el desempeño en área de trabajo, también llamada competencia disciplinar extendida y 4) competencia especializada que es el ejercicio de una habilidad especializada, como en manejo de un programa computacional avanzado.

A su vez, la Asociación Nacional de Universidades e Instituciones de Educación Superior (ANUIES, 2013) reconoce que a través de la Educación Basada en Competencias se busca vincular el sector productivo con el sistema educativo, a fin de unir los diferentes niveles de la educación (básico y medio superior) con la educación superior para que exista una coherencia y articulación con el sector productivo fundamentándose en un currículum apoyado en las competencias de manera integral y en la resolución de problemas.

Con respecto a los saberes digitales, Ramírez-Martinell y Casillas (2014) han definido diez, los cuales para esta investigación se armonizaron con el concepto de competencia al definirlos como el conjunto de habilidades y conocimientos que deben poseer los estudiantes y que además constituyen un esquema para estudiar lo que los usuarios de sistemas digitales deben saber y saber hacer con las TIC, dichos saberes están organizados en cuatro rubros: Manejo de sistemas digitales, manipulación de contenido digital, comunicación y socialización en entornos digitales y manejo de información. En esta investigación se hizo uso de dos saberes digitales, cada uno relacionado con un atributo de las competencias genéricas de la RIEMS que tienen que ver con el uso y manejo de las TIC: saber comunicarse en entornos digitales (maneja las TIC para expresar ideas) y literacidad digital (utiliza las TIC para obtener, procesar e interpretar información) cada saber se conceptualiza a través de su definición, funciones; usos y aplicaciones basándose en las hojas de saberes digitales propuesta por Ramírez-Martinell y Casillas (2014).

Saber comunicarse en entornos digitales de acuerdo con Ramírez-Martinell y Casillas (2014) se refiere a los conocimientos $\mathrm{y}$ habilidades para transmitir o recibir información (voz, mensajes de texto, fotos o videollamadas) de manera sincrónica (llamada, videoconferencia o chat) o asincrónica (correo electrónico, mensajes de texto, correo de voz) y cuyos usos y aplicaciones pueden ser: bases de datos especializadas (science direct); configuración de un perfil (Facebook, Twitter); configuración de la cuenta (Gmail, Facebook, Twitter); y videollamadas (Skype, Facetime) con fines académicos y no académicos.

A su vez, la literacidad digital se refiere a los conocimientos, habilidades y actitudes relacionadas con la búsqueda efectiva y manejo de contenido digital, mediante la consideración de palabras clave y metadatos; adopción de una postura crítica (consulta en bases de datos especializadas, búsquedas avanzadas); aplicación de estrategias determinadas (uso de operadores booleanos, definición de filtros); y consideraciones para un manejo adecuado de la información (referencias, 
difusión, comunicación) (Ramírez-Martinell y Casillas: 2014: 10), y cuyos usos y aplicación pueden ser bases de datos especializadas como science direct, buscadores avanzados como google scholar y programas para manejo de referencias bibliográficas como Zotero o EndNote.

El último de los conceptos principales de la investigación es brecha digital, Crovi (2010) por ejemplo, afirma que esta sucede en el plano tecnológico y en el plano cognoscitivo. En el primer plano menciona que la tecnología disponible no es la misma para todos ya que existen distintas condiciones para acceder a ella, lo cual pudiera estar influenciado por factores como el socioeconómico y en el plano cognoscitivo la autora se refiere a la falta de habilidades para manejar las innovaciones tecnológicas sumadas a un escaso capital cultural preexistente entre sujetos.

En el mismo sendero Claro, Espejo, Jara, y Trucco (2011) aseguran que la brecha digital en el hogar puede resolverse a través de las escuelas públicas, siempre y cuando en ellas se promueva el uso de las TIC como instrumentos de aprendizaje; Jara (2008) adiciona que es necesaria la participación del gobierno a través de su política de TIC en educación para dotar a las instituciones educativas de computadoras con Internet.

Ramírez-Martinell, Morales y Olguín (2013) en el marco del proyecto Brecha Digital caracterizan a la brecha digital como intrainstitucional y de apropiación y afirman que esta puede presentarse entre profesores; estudiantes; y profesores $\mathrm{y}$ estudiantes, considerando cuatro dimensiones: apropiación tecnológica, capital cultural, trayectoria escolar de los estudiantes y desempeño académico de los profesores.

\section{DESCRIPCIÓN}

Se desarrolló un estudio cuantitativo de tipo descriptivo a través de la aplicación del cuestionario para estudiantes diseñado en el marco del proyecto "Brecha Digital entre profesores y estudiantes de la Universidad Veracruzana: capital cultural; trayectorias escolares o desempeño académico; y grado de apropiación tecnológica GAT" que se lleva a cabo en el Centro de Investigación e Innovación en Educación Superior de la Universidad Veracruzana y que será mencionado a lo largo del documento como "Brecha Digital"

Para seleccionar la población de estudiantes los criterios de elegibilidad fueron pertenecer al Sistema Nacional de Bachillerato (SNB) en Xalapa, México en alguno de los tres subsistemas: Bachillerato General, Bachillerato Tecnológico y Profesional Técnico. Los planteles seleccionados fueron el Colegio de Bachilleres del Estado de Veracruz N’35 "Leonardo Pasquel" (Bachillerato General), el Centro de Educación Tecnológica Industrial y de Servicios N¹34 "Manuel Mier y Terán" (Bachillerato Tecnológico) y el Colegio Nacional de Educación Profesional Técnica No162 "Manuel Rivera Cambas" (Profesional Técnico).

Cabe destacar que estos planteles son los únicos ubicados en la Región Xalapa que de acuerdo con el Consejo para la Evaluación de la Educación del tipo Medio Superior están adscritos al SNB con un mismo nivel de concreción, es decir, se encuentran implementando una Educación Basada en Competencias en un mismo nivel y de acuerdo con los objetivos planteados en la RIEMS que propone un perfil de egreso único en la misma igualdad de condiciones.
Utilizando los criterios anteriores, la población quedo constituida por 547 estudiantes de la generación 2012-2015 que corresponde a todos los estudiantes de los tres planteles que se encontraban en el quinto semestre en el periodo agosto 2014enero 2015. La recolección de datos de este estudio se llevó a cabo a través del cuestionario para estudiantes elaborado en el proyecto de "Brecha digital entre estudiantes y profesores de la Universidad Veracruzana: Capital cultural; trayectorias escolares y desempeño académico; y grado de apropiación tecnológica" que recaba datos sobre apropiación tecnológica y el desarrollo de los saberes digitales de los estudiantes (Ramírez, Casillas y Ojeda, 2013). Además, con base en Zavala (2016), este cuestionario posee una fiabilidad interna de 0.961 , por lo tanto, los resultados obtenidos del cuestionario son aceptables para la investigación.

El instrumento se aplicó de manera física en siete sesiones a 194 estudiantes del bachillerato general, 206 estudiantes del bachillerato tecnológico y 147 estudiantes del profesionaltécnico. Las variables medidas se clasificaron en dos dimensiones: características del bachillerato en donde la variable independiente a medir es el Subsistema de bachillerato y los dos saberes digitales la variable dependiente.

El análisis cuantitativo de los datos se realizó a través del software especializado Statistical Package for the Social Sciences (SPSS) versión 22 el cual lleva a cabo estadística descriptiva e inferencial. Posteriormente se categorizó en cuartiles la variable dependiente y se analizó cada uno de ellos con la finalidad de obtener 4 grupos o perfiles de los estudiantes del SNB Región Xalapa, Veracruz: Bajo, Bajo-Medio, MedioAlto y Alto y se cruzaron con la variable independiente subsistema de bachillerato para describir el nivel de comunicación en entornos digitales y literacidad digital por subsistema tal como se planteó en los objetivos específicos de la investigación; por último, se realizó un análisis de medias y varianzas ANOVA a los dos saberes digitales de los estudiantes por subsistema de bachillerato para compararlo e indagar si era similar o existían diferencias significativas en los estudiantes de cada subsistema.

\section{Resultados}

Con el fin de analizar el uso y manejo de TIC de los estudiantes a través del saber comunicarse en entornos digitales y; literacidad digital se procedió a categorizarlos en cuatro niveles definidos por cuartiles: Bajo, Bajo-Medio, Medio-Alto y Alto. Quedando entonces los intervalos de cada nivel de la siguiente manera:

\section{Tabla I}

\begin{tabular}{|c|c|}
\hline \multicolumn{2}{|c|}{ Nivel de manejo de TIC } \\
\hline Perfil & Intervalo \\
\hline Bajo & $2.87-4.68$ \\
\hline Bajo-Medio & $4.69-5.27$ \\
\hline Medio-Alto & $5.28-5.90$ \\
\hline Alto & $5.91-7.77$ \\
\hline
\end{tabular}

Las medias del saber digital "Comunicarse en entornos digitales" fueron muy parecidas entre sí, siendo el Bachillerato General quien presenta una media mayor de 4.94 casi a la par 
con el Profesional-Técnico con 4.92 seguidos del Bachillerato Tecnológico con 4.73. El valor máximo en los tres subsistemas fue de nueve y uno el valor mínimo en los subsistemas

Tabla 2

Medias de

Comunicación en entornos digitales

\begin{tabular}{|l|l|l|l|l|l|}
\hline $\begin{array}{l}\text { Subsistema } \\
\text { de Bach. }\end{array}$ & Frec. & Mín. & Máx. & Media & Nivel \\
\hline $\begin{array}{l}\text { Profesional- } \\
\text { Técnico }\end{array}$ & 144 & 1 & 9 & 4.92 & $\begin{array}{l}\text { Bajo- } \\
\text { Medio }\end{array}$ \\
\hline General & 199 & 2 & 9 & 4.94 & $\begin{array}{l}\text { Bajo- } \\
\text { Medio }\end{array}$ \\
\hline Tecnológico & 206 & 1 & 9 & 4.73 & $\begin{array}{l}\text { Bajo- } \\
\text { Medio }\end{array}$ \\
\hline
\end{tabular}

Tecnológico y Profesional-Técnico en una escala del 1 al 10 y situándose los tres grupos en el nivel Bajo-Medio

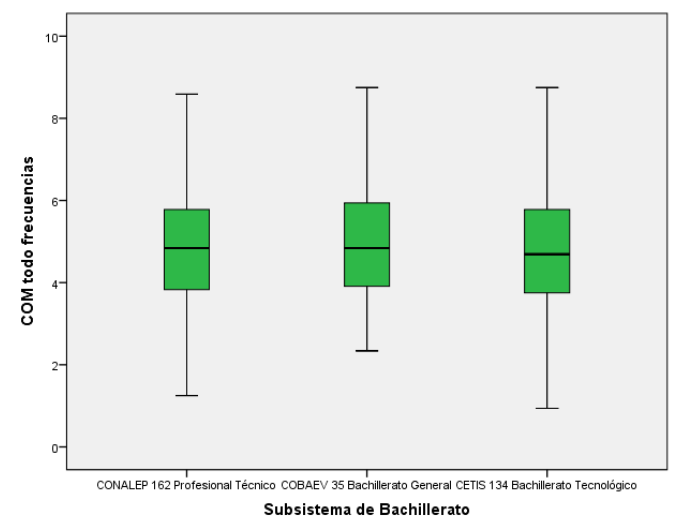

Figura 1. Comunicación por subsistema de bachillerato

Por su parte, las medias de Literacidad Digital también tuvieron diferencias poco significativas y fueron muy similares, siendo el Bachillerato General quien presenta una media mayor de 7.21, seguida del Profesional-Técnico con 6.81para finalizar con el Bachillerato Tecnológico con 6.72. El valor máximo en los tres subsistemas fue de diez y el valor mínimo fue de 4 en el Bachillerato Tecnológico y Profesional-Técnico en una escala del 1 al 10 y situándose los tres grupos en el nivel Alto.

Tabla 3

Medias de

Literacidad digital

\begin{tabular}{|l|l|l|l|l|l|}
\hline $\begin{array}{l}\text { Subsistema } \\
\text { de Bach. }\end{array}$ & Frec. & Mín. & Máx. & Media & Nivel \\
\hline $\begin{array}{l}\text { Profesional- } \\
\text { Técnico }\end{array}$ & 141 & 4 & 10 & 6.81 & Alto \\
\hline General & 188 & 5 & 10 & 7.21 & Alto \\
\hline Tecnológico & 189 & 4 & 10 & 6.72 & Alto \\
\hline \multicolumn{7}{|c|}{ Fuente: Elaboración propia } \\
\hline
\end{tabular}

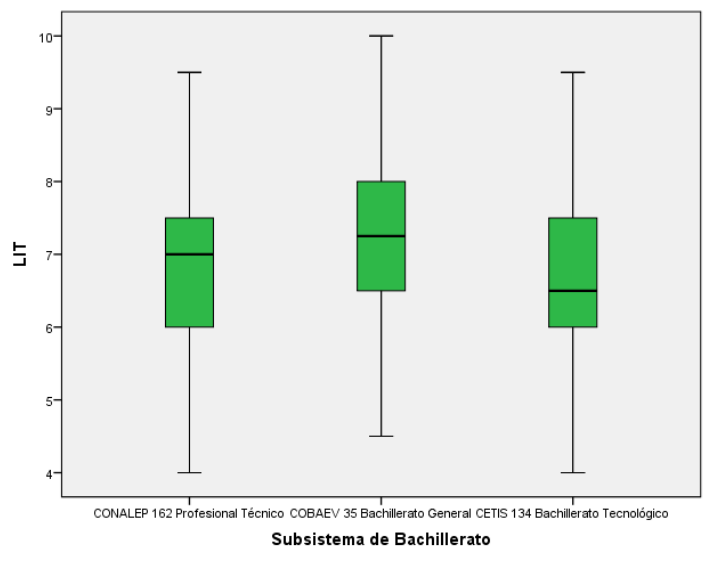

Figura 2. Literacidad digital por subsistema de bachillerato

\section{CONCLUSIONES}

Tras el estudio realizado, se cumplieron los objetivos planteados al inicio de este, al analizar de manera comparativa el nivel de uso y manejo de TIC como competencia genérica en los estudiantes del Sistema Nacional de Bachillerato Región Xalapa, Veracruz para indagar si era igual en tres subsistemas de bachillerato.

Para cumplir con los objetivos específicos se describió el nivel de comunicación en entornos digitales de los estudiantes del Sistema Nacional de Bachillerato en Xalapa, Veracruz por subsistema de bachillerato (general, tecnológico y profesional técnico) y al no encontrarse diferencias significativas se concluye que los estudiantes de los tres subsistemas poseen un mismo nivel cognitivo e instrumental de este saber digital situándose los tres en un nivel Bajo-Medio.

Por su parte y cumpliendo con el siguiente objetivo específico se describió el nivel de dominio de literacidad digital de los estudiantes del Sistema Nacional de Bachillerato en Xalapa, Veracruz por subsistema de bachillerato (general, tecnológico y profesional técnico) y al no encontrarse diferencias significativas se concluye que los estudiantes de los tres subsistemas poseen un mismo nivel cognitivo e instrumental de este saber digital situándose en un nivel Alto.

Con lo anterior, se puede concluir que los estudiantes de los tres subsistemas de bachillerato en la ciudad de Xalapa en México a pesar de poseer niveles de dominio similares entre subsistemas en los dos saberes digitales relacionados con los atributos de las competencias genéricas de la RIEMS lo han desarrollado en diferentes dominios ya que mientras se comunican en entornos digitales en un nivel Bajo-Medio de acuerdo con los hallazgos descritos anteriormente pueden obtener, procesar e interpretar información en un nivel Alto.

A pesar de no existir diferencias significativas, se puede apreciar que los estudiantes del bachillerato general se localizan ligeramente más arriba que los estudiantes de los otros dos subsistemas en ambos saberes digitales, mientras que los estudiantes del subsistema tecnológico son los que obtuvieron menores puntajes en ambos saberes.

Resulta importante que los estudiantes preuniversitarios próximos a ingresar a la ES posean habilidades digitales, las instituciones educativas en México deben entonces trabajar de manera coordinada para seguir desarrollando competencias genéricas en el uso y manejo de las TIC. 


\section{REFERENCIAS}

ANUIES (2013) Anuario estadístico de Educación Media Superior. Recuperado de http://www.anuies.mx/content.php?varSectionID=166

Cervantes, J. (2015) La mediación pedagógica a través de las TIC. Una propuesta para la formación docente en Educación Media Superior (Tesis de Maestría) Universidad Pedagógica Nacional, México. Recuperado de http://xplora.ajusco.upn.mx:8080/xplorapdf/30816.pdf

Claro, M., Espejo, A., Jara, I. y Trucco, D. (2011). Aporte del sistema educativo a la reducción de las brechas digitales. Una mirada desde las mediciones PISA. Chile: CEPAL.

Crovi Druetta, D. (2010). Jóvenes, migraciones digitales y brecha tecnológica. Revista Mexicana de Ciencias Políticas y Sociales. LII (209), 119-133. UNAM. México

Jara Valdivia, I. (2008). Las políticas de tecnología para escuelas en América Latina y el mundo: visiones y lecciones. Chile: CEPAL.

Ramírez-Martinell, A., Casillas, M. A. y Ojeda, M. (2013). Brecha digital entre estudiantes y profesores de la Universidad Veracruzana: Capital cultural; trayectorias escolares y desempeño académico; y grado de apropiación tecnológica. Recuperado de http://www.uv.mx/blogs/brechadigital/files/2013/08/proy ecto brecha_digital_2013 11.pdf

Ramírez Martinell, A., Morales, A.T., y Olguín, P.A., (2013) Brecha Digital en el contexto universitario: Una estrategia para su medición. Memorias del XII Congreso Nacional de Investigación Educativa, Guanajuato. México

Ramírez-Martinell, A. y Casillas, M. (2014) Hojas de trabajo de los saberes digitales. Blog del proyecto de Brecha Digital en Educación Superior [Mensaje en un blog]. Recuperado de

http://www.uv.mx/blogs/brechadigital/2014/08/24/hojas saberes_digitales/

Secretaría de Educación Pública [SEP] (2008) Acuerdo Número 442, por el que se establece el Sistema Nacional de Bachillerato en un marco de diversidad. Diario Oficial de la Federación. Recuperado de http://www.sep.gob.mx/work/models/sep1/Resource/7aa 2c3ff-aab8-479f-ad93-db49d0a1108a/a442.pdf

Secretaría de Educación Pública [SEP] (2008b) Acuerdo Número 444, por el que se establece el Sistema Nacional de Bachillerato en un marco de diversidad. Diario Oficial de la Federación. Recuperado de http://www.sep.gob.mx/work/models/sep1/Resource/7aa 2c3ff-aab8-479f-ad93-db49d0a1108a/a444.pdf

Tovar, R. y Serna, G. (2013) 332 estrategias para educar por competencias. Cómo aplicar las competencias en el aula para bachillerato. México: Trillas

Zavala Alcalá, Z. (2016). El grado de apropiación tecnológica y el rendimiento académico de los estudiantes universitarios. Tesis de maestría. Instituto de Investigaciones en Educación. Universidad Veracruzana. México. 